\title{
Inspiraling Black Holes: The Close Limit
}

\author{
Gaurav Khanna, ${ }^{1}$ John Baker, ${ }^{1,2}$ Reinaldo J. Gleiser, ${ }^{3}$ Pablo Laguna, ${ }^{4}$ Carlos O. Nicasio, ${ }^{3}$ \\ Hans-Peter Nollert, ${ }^{5}$ Richard Price, ${ }^{6}$ and Jorge Pullin ${ }^{1}$ \\ ${ }^{1}$ Center for Gravitational Physics and Geometry, Department of Physics, The Pennsylvania State University, \\ 104 Davey Lab, University Park, Pennsylvania 16802 \\ ${ }^{2}$ Max-Planck-Institut für Gravitationsphysik, Albert-Einstein-Institut, Am Mühlenberg 1, D-14476 Golm, Germany \\ ${ }^{3}$ Facultad de Matemática, Astronomía y Física, Universidad Nacional de Córdoba, Ciudad Universitaria, \\ 5000 Cordoba, Argentina \\ ${ }^{4}$ Department of Astronomy and Astrophysics, The Pennsylvania State University, \\ 525 Davey Lab, University Park, Pennsylvania 16802 \\ ${ }^{5}$ Theoretische Astrophysik, Universität Tübingen, 72076, Tübingen, Germany \\ ${ }^{6}$ Department of Physics, University of Utah, Salt Lake City, Utah 84112
}

(Received 21 May 1999)

\begin{abstract}
We calculate an estimate of the gravitational radiation emitted when two equal mass black holes coalesce at the end of their binary inspiral, using several approximations based on considering the holes close to each other. A shortcoming of our method is that it is limited to models forming final holes with slow rotation, but our results clearly suggest a trend for larger angular momenta. We find that about $1 \%$ of the mass energy of the pair will emerge as gravitational waves during the final stages of the collision and that a negligible fraction of the angular momentum will be radiated.
\end{abstract}

PACS numbers: 04.30.Db, 04.25.Dm, 04.70.Bw, 97.80.Fk

An international network of interferometric gravitational wave $(\mathrm{GW})$ observatories (the LIGO project in the U.S., the VIRGO and GEO projects in Europe, and the TAMA project in Japan [1]) will be capable of detecting gravitational waves in the next few years. This could have revolutionary implications for astronomy since it constitutes a new form of "light" with which to observe the Universe, a form that is better correlated with the bulk motions of matter and is very hard to shield or distort. Gravitational waves were originally predicted by Einstein in 1915 but their theoretical existence was not completely understood until the 1960's [2] and their direct experimental detection has remained a daunting challenge [3]. One of the most promising sources for detection is the collision of two black holes to form a single, final hole. Such a collision is expected to be the end point of the decaying inspiral for a binary pair of holes. Just how promising such collisions are depends very much on the masses of the colliding holes and is connected with the characteristics of detectors. The sensitivity of the laser interferometric detectors, like LIGO, peaks around $100 \mathrm{~Hz}$.

The major determinant of the frequency of GWs produced in a black hole inspiral/collision is $M$, the mass of the system (and of the final black hole formed). The frequency scales as $1 / M$ and is around $10^{4} \mathrm{~Hz}$ for a $1 M_{\odot}$ system. The usual black hole candidates are stellar mass holes and supermassive holes $\left(\sim 10^{6} M_{\odot}\right)$ in the centers of many galaxies. The frequency of GWs from the former candidates would be too high except for the weak radiation from the early inspiral. GWs from supermassive holes would be too low in frequency for Earth based systems but well suited to space based detection. Black holes of $100 M_{\odot}$ would be ideal as sources and black holes of any mass can in principle exist. But the astrophysical motivation for such "middleweight" holes was weak. Recent observations [4] of x-ray emission from galaxies suggest that some galaxies may contain middleweight holes, perhaps as an evolutionary stage in the formation of supermassive holes. Should such a middleweight hole exist it will produce GWs near the optimal $\sim 100 \mathrm{~Hz}$ frequency when it collides with compact objects of equal or smaller masses.

For two different reasons, we focus here on the collision of roughly equal mass holes. The first is that the power radiated from the collision of holes of masses $m_{1} \leq m_{2}$ scales as $\left(m_{1} / m_{2}\right)^{2}$, at least in the case that $m_{1}$ is significantly smaller than $m_{2}$. The GW strain produced in a detector will then scale as $m_{1} / m_{2}$, and collisions of equal mass holes will be the most detectable. The second reason is that computations in the case $m_{1} \ll m_{2}$ can be done by treating the smaller mass as a particle perturbing the spacetime of the hole. By contrast, even a qualitative understanding is lacking for the collisions of equal mass holes at the end point of binary inspiral. It is not known, for example, whether the inspiraling holes will smoothly and gradually merge to form a single final hole or whether the smooth inspiral will end with a plunge of the holes into each other. It is commonly accepted that numerical solutions of Einstein's field equations, on supercomputers, will be a necessary element in understanding such collisions. Although numerical relativity has given a fairly complete picture of head-on collisions, numerical studies of the astrophysically interesting case, truly three dimensional collisions, are proving to be remarkably difficult. Progress with this problem is characterized by approximate and imperfect results. We present here such a 
result: the first approximate calculation for the gravitational radiation emitted by the collision at the end of the inspiral of two equal mass holes. We find, in particular, that only about $1 \%$ of the mass of the two hole system will be radiated in the collision. The several approximations that limit the generality of this conclusion will be spelled out below.

The radiation of interest is generated at the end of the slow quasi-Newtonian binary inspiral, when the interaction between the holes becomes highly nonlinear. It is useful to break down the subsequent evolution of the binary into two stages: the "merger" describing the transition from two disjoint holes to a single final, highly distorted hole; and the "ringdown" stage in which the final hole relaxes to a stationary final state by the emission of GWs. Here we will consider only GW radiation during the latest stage of coalescence (ringdown and approach to ringdown). In particular, we use the close limit method in which we treat the late stage evolution as a perturbation of the final hole. The evolution can then be computed with the relatively simple linear equations of perturbation theory, rather than the nonlinear equations of Einstein's full theory. To evolve a spacetime in general relativity, one needs to provide initial data, a 3-geometry $g_{a b}$ and an extrinsic curvature $K_{a b}$, that solve Einstein's equations on some starting hypersurface (i.e., at some starting time). For two black holes, this is an easy task if the holes are far apart, since one can superpose the solutions for two individual holes ignoring their interactions. When the black holes are close on the initial hypersurface, the astrophysically correct initial data are the solution corresponding to what would have evolved during the binary inspiral, but such an evolution cannot be computed with present day techniques. One must therefore use a somewhat artificial initial data solution that is a best guess at a representation of close black holes. The need for such a guess is one of the sources of uncertainty in our result. It should be noted that the choice of initial data is not a shortcoming if our answers are to be used as code checks for numerical relativity, since the same initial data can be used for our close limit evolution and in numerical relativity codes.

A convenient prescription for initial data has been given by Bowen and York [5] and is used in much of numerical relativity. Their method assumes that the initial spatial geometry metric $g_{a b}$ is conformal to a flat space according to $g_{a b}=\phi^{4} \delta_{a b}$. One specifies the location of any number of holes in the conformal flat space and specifies parameters representing the momentum and spin of each hole. In the limit that the holes are far from each other on the initial hypersurface, these parameters have the usual physical meaning. When the holes are close, the "separation," "spin," and "momentum" of an individual hole has no unambiguous meaning. It would seem a priori that any evolution done with uncertain initial data is of little value. But a large set of examples [6] computed for head-on collisions, and compared with numerical relativity results, gives us a basis for considering that our predictions, based on "reasonable" initial data choices, have a certain degree of validity, at the very least as order of magnitude estimates, but more likely as correct within a factor of 2 or so.

In the Bowen-York formalism the extrinsic curvature for a hole $i$ with no spin, and with momentum $\vec{P}^{(i)}$, is $K_{a b}=\phi^{-2} \hat{K}_{a b}$, where

$$
\hat{K}_{a b}^{(i)}=\frac{3}{2 R_{(i)}^{2}}\left[2 P_{(a}^{(i)} n_{b)}^{(i)}-\left(\delta_{a b}-n_{a}^{(i)} n_{b}^{(i)}\right) P_{(i)}^{c} n_{c}^{(i)}\right] .
$$

Here $R_{(i)}$ and $\vec{n}^{(i)}$ are respectively the distance to hole $i$, and the direction from the hole, in the conformal flat space. In Eq. (1) and below we use units in which $G=c=1$. Our choice of initial data is a Bowen-York specification that most plausibly represents a binary pair of nonspinning holes. In particular our initial data are taken to represent equal mass holes at $x= \pm L / 2$ in the conformal flat space. The holes are taken to have momenta $\vec{P}^{(1)}$ and $\vec{P}^{(2)}$ that have equal magnitude $P$, and that have opposite directions orthogonal to the line joining the holes. The equations satisfied by $\hat{K}_{a b}$ are linear and independent of $\phi$, so for the two hole system we can take the extrinsic curvature to be that given by the sum $\hat{K}_{a b}=\hat{K}_{a b}^{(1)}+\hat{K}_{a b}^{(2)}$ of the contributions of each of the holes. For small $L$, the directions $n^{(i)}$ and distances $R_{(i)}$ to the two holes are the same up to corrections of order $L / r$. Since the two momenta are in opposite directions, the total extrinsic curvature $\hat{K}_{a b}$ vanishes as $L \rightarrow 0$.

The conformal factor $\phi$ is required to satisfy a nonlinear elliptical equation $\nabla^{2} \phi=-K_{a b} K^{a b} / 8 \phi^{7}$ that is not analytically solvable. To get a closed form expression for $\phi$, we use the "slow" approximation: For small $P$ the product $K_{a b} K^{a b}$ is second order in $P$ and one can approximate the solution by a solution of $\nabla^{2} \phi=0$. Solutions of the latter with the topology of two holes are well known and it is known that this approximation works better than might be expected [6,7]. For larger values of the momenta, $K_{a b}$ grows linearly whereas $\phi$ grows as a fractional power of the momenta. Therefore for larger values the extrinsic curvature dominates the initial data and the error we are making in the determination of the conformal factor $\phi$ is again negligible. This was explicitly verified in a study of the head-on collisions of boosted black holes [6,7].

With the choice of $\hat{K}_{a b}$ and approximate solution for $\phi$ the specification of initial data is complete. In the limit that $L \rightarrow 0$, the initial data are those of a stationary hole, so we can view $P, L$, or $P L$ as an expansion parameter, and we can evolve forward in time using perturbation theory. It would seem that this perturbative evolution should be done with the Teukolsky formalism [8] for perturbations of Kerr holes, since the final hole formed in binary inspiral is expected to be a rapidly rotating (i.e., Kerr) hole, perhaps possessing angular momentum $J$ that 
is near the extreme Kerr limit $J / M^{2}=1$ for black holes. It turns out, however, that the initial data described above are not a perturbation of a Kerr hole. In the space of parameters $P$ and $L$, there is no family of solutions, with fixed nonzero angular momentum, that has a Kerr hole as the zero perturbation limit. This can be viewed as a consequence of the very constraining requirements that must be satisfied by Kerr initial data [9]. It is therefore improper to consider the spacetime evolving from the initial data to be a "small departure" from a Kerr hole.

We can consider the initial data to be perturbations of a nonrotating (Schwarzschild) hole. The initial data are purely even parity and we use the perturbation formalism developed by Zerilli [10], in which even parity perturbations are decomposed in angular multipoles. As is usually the case, radiation is dominantly quadrupolar. The two hole data have $\ell=2$ radiation for $m=0, \pm 2$. For example, the Zerilli function $\psi_{(2,2)}$, describing the $\ell=2, m=2$ multipole, has the Cauchy data

$$
\begin{aligned}
\psi_{(2,2)}(0, r)= & 4 \sqrt{\frac{2 \pi}{15}} M L^{2} \\
& \times \frac{r(7 \sqrt{r}+5 \sqrt{r-2 M})}{(2 r+3 M)(\sqrt{r}+\sqrt{r-2 M})^{5}}, \\
\dot{\psi}_{(2,2)}(0, r)= & -\frac{i \sqrt{30 \pi}}{10} P L \frac{(4 r+3 M) \sqrt{r-2 M}}{r^{5 / 2}(2 r+3 M)},
\end{aligned}
$$

and the Zerilli function for $(\ell=2, m=-2)$ is the complex conjugate of $\psi_{(2,2)}(t, r)$.

It will be very important to know the range of validity of our perturbative result, that is, the maximum value of $P L$, or angular momentum, for which our results are reasonably accurate. This question is equivalent to the question "how early in the coalescence can the close limit approximation be used?" To answer this, we rely on experience with computations of head-on collisions, where comparisons were available with numerical results for fully nonlinear evolutions. A more objective approach would be to compute answers to second order in the perturbation, as has been done successfully for head-on collisions [6]. Since our present approach is limited by the use of initial data that would make our predictions only roughly accurate, the very lengthy computations of second-order theory do not seem justified at the present time. Instead, as a "poor person's" approach to estimating the error of our calculations, we have also carried out the evolution of the spacetime using the Teukolsky formalism. For each choice of the angular momentum perturbation $P L$, we have taken the initial data to specify a perturbation of a Kerr spacetime with angular momentum parameter $a=P L / M$. (This appears at first intuitively reasonable, but we have argued already that a nonrotating hole is really the correct background.) In this hybrid approach, a change in the parameter $P L$ does not take us along a family of perturbations of a single spacetime, but the result of the calculation will be "first order" in $P L$ in some sense. The difference between the result of Teukolsky evolution and Zerilli evolution, therefore, gives us a crude indicator of the limit of validity of first-order perturbation theory.

The curve on the left in Fig. 1 shows the waveform of the gravitational radiation, that is, the strain that would affect the arm lengths of an interferometer. The curves on the right show the total energy carried in the
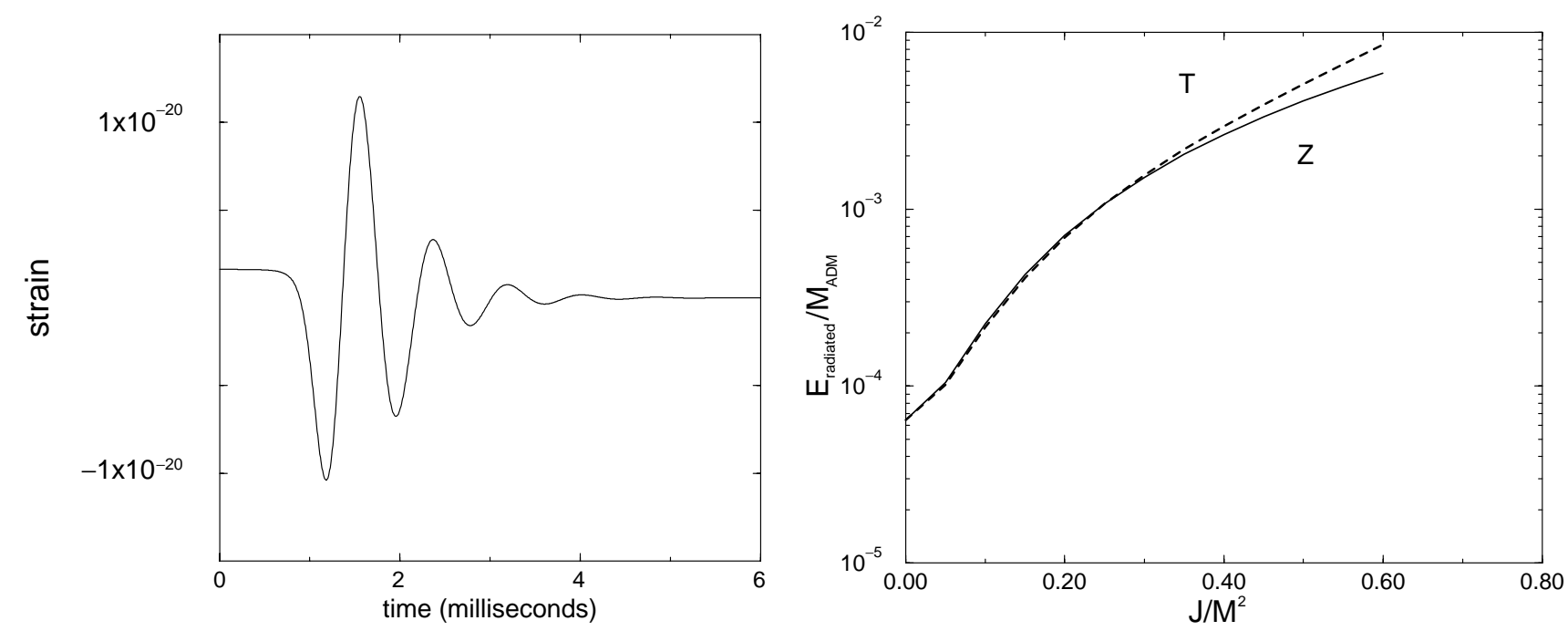

FIG. 1. The panel on the left shows the strain amplitude in the equatorial plane as a function of time, produced by a $10 M_{\odot}$ black hole binary going through its ringdown phase at a distance of $100 \mathrm{Mpc}$ from the detector. We assume that the detector is oriented for maximum sensitivity to the radiation in the orbital plane. (For an L-shaped laser interferometer, one arm of the L would have to have the orientation just described for the bar; the other arm would have to be perpendicular to the orbital plane.) The right panel shows the fraction of the mass of the system radiated as gravitational waves as a function of the normalized initial angular momentum of the collision. 
waves as a function of the expansion parameter $\epsilon=$ $J / M^{2}$. The result shown is for two black holes initially separated in conformal flat space by $L=1.8$ in terms of the mass of each hole. (If one were considering a Misner-type geometry, the proper separation measured along the geodesic threading the throats would be 5.5 in the same units [11].) The curve labeled $Z$ shows results for linearized perturbation calculations using the Zerilli equation; the curve $T$ shows the result of "hybrid perturbation" calculations using the Teukolsky equation. The two results diverge around parameter values of $\epsilon=$ 0.4 to 0.5 , and this is a reasonable limit to take for the applicability of perturbation estimates. We note that the Teukolsky results lie above the Zerilli results, and this weakly suggests that the Zerilli-based estimates are more accurate. (In close limit estimates for head-on collisions, linearized results always overestimated the nonlineari.e., numerical relativity - results.)

The limitation to $\epsilon$ less than or around 0.5 was expected but is unfortunate, since most realistic collisions will probably take place with angular momentum closer to $\epsilon=1$ [12]. One can make an educated guess of what could happen around $\epsilon \sim 1$ by looking at the curves in Fig. 1, and by extrapolating by eye to $\epsilon \sim 1$. It is difficult in this way to make a case for radiation more than $1 \%$ of the system's mass. This is in rough agreement with the $3 \%$ value used in data analysis calculations of Flanagan and Hughes [12].

The radiated angular momentum is well defined in linear perturbation theory even for spacetimes without any symmetry. It can be computed in two very different ways, either from an angular momentum flux constructed from the Landau-Lifshitz pseudotensor or by looking at the change in the $\ell=1$ odd parity perturbation. In either case we find that the radiated angular momentum is given by (after a somewhat lengthy calculation similar to that carried out in [13] for the radiated energy)

$$
\begin{aligned}
\Delta J=\frac{3}{2 \pi} \lim _{r \rightarrow \infty} \int_{0}^{-\infty} & {\left[\operatorname{Im}(\psi) \frac{\partial \operatorname{Re}(\psi)}{\partial t}\right.} \\
& \left.-\operatorname{Re}(\psi) \frac{\partial \operatorname{Im}(\psi)}{\partial t}\right] d t,
\end{aligned}
$$

where we have written $\psi_{(2,2)}(t, r)=\operatorname{Re}(\psi)+i \operatorname{Im}(\psi)$. For values of $\epsilon$ from around 0.1 to 0.5 , the angular momentum radiated is roughly $0.1 \%$ of the initial angular momentum. For a given multipole with azimuthal index $m$, and single frequency $\omega$, the angular momentum radiated divided by the energy radiated will be less than or equal to $|m / \omega|[12,14]$. If we take $\omega$ to be the real part of the frequency of the least damped, $\ell=2$, quasinormal mode, and we take $m=2$, this tells us that the ratio of $E / M$ radiated to $J / M^{2}$ radiated should be no greater $\sim 0.2$, and this is approximately the case near $\epsilon=0.5$. That is, there is very little angular momentum radiated, but this is to be expected on general grounds, given the amount of energy radiated, following the arguments of $[12,14]$.

In summary, we have used the "close limit" to estimate the radiation in the collision at the end of the inspiral of two equal mass nonrotating black holes. The assumptions and restrictions were the following: (i) only the "latestage" radiation was computed; (ii) we assumed that a simple initial data set gave an adequate representation of appropriate astrophysical conditions; (iii) we assumed that the final hole is not near the extreme Kerr limit; (iv) we used close limit estimates of the evolution. Our main conclusion is that the energy radiated in late stage is probably not more than $1 \%$ of the total mass of the system. The most serious uncertainty in this result is the possibility that the radiation from the early merger stage of coalescence is very much larger than the late stage radiation. With our $1 \% M c^{2}$ estimate, collisions of black holes of $100 M_{\odot}$ would be detectable with signal to noise of 6 out to distances on the order of $200 \mathrm{Mpc}$ by the initial LIGO configuration and to distances of $4 \mathrm{Gpc}$ with the advanced LIGO detector.

This work was supported in part by Grants No. NSFINT-9512894, No. NSF-PHY-9357219, No. NSFPHY-9423950, No. NSF-PHY-9734871, No. NSF-PHY9800973, No. PHY-9407194, No. NATO-CRG971092, No. DFG-SFB-382, by funds of the University of Córdoba, the University of Utah, and Penn State. We also acknowledge support of CONICET and CONICOR (Argentina). J.P. also acknowledges support from the Alfred P. Sloan and the John S. Guggenheim foundations.

[1] For current information and links to all the interferometric gravitational wave detector projects, see http://www.ligo.caltech.edu.

[2] D. Kennefick, gr-qc/9704002.

[3] P. Saulson, Fundamentals of Interferometric Gravitational Wave Detectors (World Scientific, Singapore, 1994).

[4] A. Ptak and R. Griffiths, astro-ph/9903372; E. Colbert and R. Mushotzky, astro-ph/9901023.

[5] J. Bowen and J. York, Phys. Rev. D 21, 2047 (1980).

[6] O. Nicasio, R. Gleiser, R. Price, and J. Pullin, Phys. Rev. D 59, 044024 (1999).

[7] J. Baker, A. Abrahams, P. Anninos, S. Brandt, R. Price, J. Pullin, and E. Seidel, Phys. Rev. D 55, 829 (1997).

[8] S. Teukolsky, Astrophys. J. 185, 635 (1973).

[9] W. Krivan and R. Price, Phys. Rev. D. 58, 104003 (1998).

[10] F. J. Zerilli, Phys. Rev. D 2, 2141 (1970).

[11] See, for instance, P. Anninos, R. Price, J. Pullin, E. Seidel, and W.-M. Suen, Phys. Rev. D 52, 4462 (1995).

[12] E. Flanagan and S. Hughes, Phys. Rev. 57, 4535 (1998); 57, 4566 (1998).

[13] C. Cunningham, R. Price, and V. Moncrief, Astrophys. J. 230, 870 (1979).

[14] K. S. Thorne, Rev. Mod. Phys. 52, 299 (1980), Eqs. (4.16) and (4.23). 